\title{
Metal-Insulator Transition and Heterostructure Formation by Glycines Self-Assembled on Defect-Patterned Graphene
}

\author{
F. Ersan, ${ }^{\dagger, \dagger}$ O. Üzengi Aktürk, ${ }^{\S, \|}$ E. Aktürk, ${ }^{*},, \|_{\odot}$ and S. Ciraci*,* \\ ${ }^{\dagger}$ Department of Physics, ${ }^{\S}$ Department of Electric and Electronic Engineering, and "Nanotechnology Application and Research \\ Center, Adnan Menderes University, Aydın 09010, Turkey \\ ${ }^{\ddagger}$ Department of Physics, Bilkent University, Ankara 06800, Turkey
}

ABSTRACT: This study unveils critical physical and chemical processes taking place at the interface between an amino acid, glycine, and defected graphene. Although glycine interacts weakly through van der Waals attraction with pristine graphene, it can set rather strong bonding at the close proximity of low-coordinated $\mathrm{C}$ atoms of single and triple vacancies and also at the edges of nanoribbons. The adsorption of a glycine molecule first leads to a reconstruction of the defect through a concerted process, which in turn induces magnetic metal-nonmagnetic insulator transition. This way, glycine can be pinned at the defect site with well-
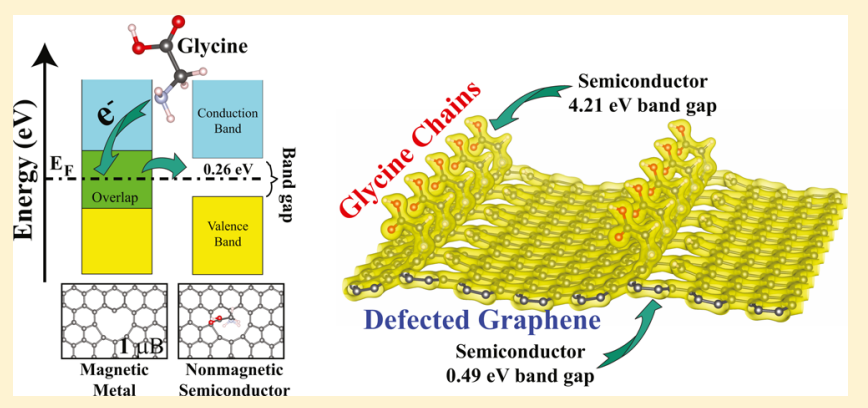
defined atomic configuration and electronic structure. In particular, libration frequency of the adsorbed glycine is attributed to significant restoring forces, which is essential for the self-assembly of glycines. Organic overlayer produced by self-assembled glycines on defect-patterned graphene constitutes a junction (heterostructure) with bilateral electronic and optical properties and offers novel device functions from biographene interfaces. These predictions are obtained from first-principles calculations using density functional theory.

\section{INTRODUCTION}

Advances in nanoscience and nanotechnology have brought the bio-two-dimensional (2D) material interface and critical physical interactions therein into focus. Recently, the study of this interface has contributed to the characterization of biomolecules and has led to the discovery of biosensors and smart drugs. The large surface area relative to their volumes is the prime reason why $2 \mathrm{D}$ materials acquired increasing role in the biointerface research. Graphene is the most popular material because of its extraordinary properties, such as high surface area, excellent mechanical strength, high carrier mobility and thermal conductivity, ${ }^{1}$ and low Johnson noise. ${ }^{2}$

Proteins being one of the most crucial ingredient of living organisms contain many amino acids. Present study on the nature of interactions of amino acids with graphene can provide critical information regarding their fixation for indepth characterization and synthesis of new peptides. Additionally, functional overlayers of amino acids self-assembled on defect-patterned graphene can be realized. Nanowires, heterostructures, and single-photon and single-electron tunneling sources can be constructed in these organic overlayers for biodevices.

Earlier experimental and theoretical studies have been performed to analyze amino acids, ${ }^{3-6}$ polyamines, steroids, anticancer drugs, and nucleosides. ${ }^{3-11}$ Among all amino acids, glycine (or Gly, $\mathrm{NH}_{2} \mathrm{CH}_{2} \mathrm{COOH}$ ) has a special place because Gly acts as an inhibitory neurotransmitter in the central nervous system in brain ${ }^{12}$ with an estimated concentration of approximately $1 \mathrm{mM}$ in the normal adult brain. ${ }^{13}$ This concentration increases in high-grade brain tumors. ${ }^{12}$ Like other amino acids, Gly also has a weak van der Waals (vdW) interaction with pristine graphene. ${ }^{7,14-19}$ Even Stone-Wales (SW) defected graphene and oxygen atom-decorated graphene give rise to only minute increase to the binding energy of Gly. ${ }^{16}$ However, it is well-known that twofold coordinated C atoms at the vacancy sites in graphene can readily interact with adspecies. $^{20-24,43}$ For example, $\mathrm{O}_{2}$ and $\mathrm{OH}$ molecules dissociate into their constituents at the vacancy site of graphene. ${ }^{21,43}$ Expectantly, low-coordinated $\mathrm{C}$ atoms in graphene can be active sites to provide relatively stronger binding of Gly to graphene.

In this paper, we investigate the interaction of Gly molecule(s) with pristine and defected graphene having single vacancy and trivacancy, as well as the edges of ribbons. The binding of Gly to pristine graphene is weak, even if it can increase slightly either with the negative charging or with the coadsorbed $\mathrm{H}_{2} \mathrm{O}$ molecules at the close proximity. However, we found that a Gly can induce reconstruction at the vacancy site via rebonding and eventually it can be pinned at the twofolded $\mathrm{C}$ atom with a sizeable binding energy. In the course of the adsorption process, the magnetic and metallic substrate can undergo a metal-insulator transition and eventually becomes

Received: April 11, 2018

Revised: June 1, 2018

Published: June 5, 2018 

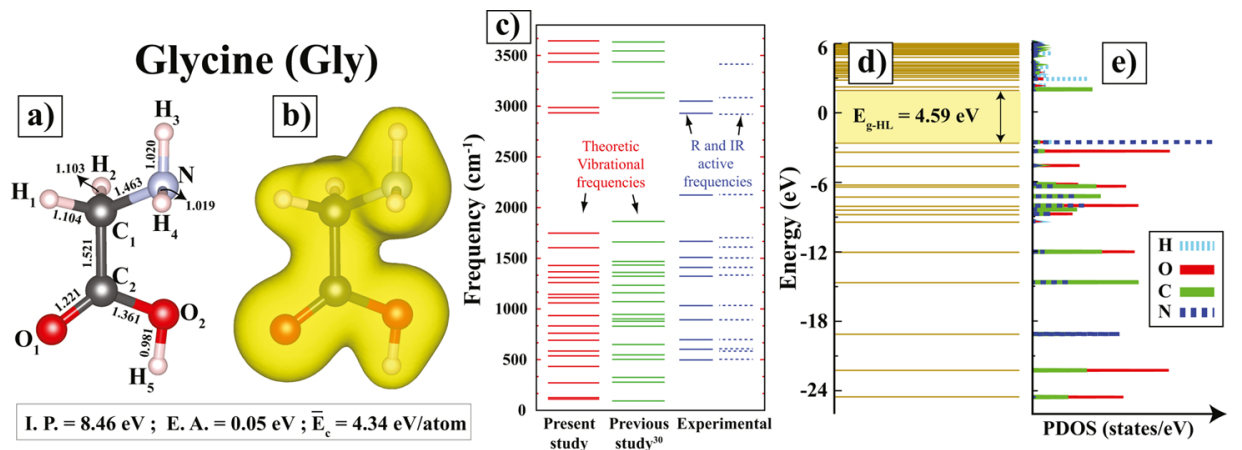

Figure 1. (a) Atomic configuration of free $\mathrm{Gly}, \mathrm{NH}_{2} \mathrm{CH}_{2} \mathrm{COOH}$, with optimized bond lengths between constituent $\mathrm{N}, \mathrm{C}, \mathrm{O}$, and $\mathrm{H}$ atoms. (b) Isosurfaces of the total charge density (isosurface level is 0.035 electrons/ $\AA^{3}$ ). (c) Calculated frequencies of vibration modes (red lines), previously calculated frequencies (green lines), experimentally measured Raman frequencies (blue solid lines), and infrared Raman frequencies ${ }^{30}$ (blue dashed lines). (d) Calculated energy-level diagram with HOMO-LUMO gap, $E_{\mathrm{g}-\mathrm{HL}}=4.59 \mathrm{eV}$. (e) Densities of states projected to constituent atoms projected density of states. Calculated values of IP, EA, and average cohesive energy are also presented.

nonmagnetic. Adsorbed Glys attain significant libration frequencies and well-defined configuration dictated by the restoring forces originating from the chemical bonding with graphene. With the self-assembly properties acquired this way, organic overlayers can be constructed on defect-patterned graphene to form composite structures with bilateral electronic properties.

\section{COMPUTATIONAL DETAILS}

First-principles spin-polarized plane-wave calculations were carried out within density functional theory using projector augmented-wave potentials ${ }^{45,46}$ and exchange-correlation potential approximated by generalized gradient approximation using Perdew, Burke, and Ernzerhof functional ${ }^{47}$ including vdW corrections in D2 level (D2-G). ${ }^{48}$ Other vdW corrections specified as D3-G ${ }^{49}$ and $\mathrm{BJ} .{ }^{50}$ were also used for the sake of comparison. Wave functions are expanded in plane-wave basis sets up to electron kinetic energy cutoff of $550 \mathrm{eV}$. A single Gly bound to the pristine graphene or to a defect site such as single vacancy or trivacancy is modeled by an $(8 \times 8)$ periodic supercell in the $x y$-plane which is stacked along the $z$-direction with a $15 \AA$ vacuum spacing to avoid interlayer interactions. Brillouin zone integration is performed with a $9 \times 9 \times 1 k$ point mesh, following the convention of Monkhorst and Pack. ${ }^{51}$ Ionic relaxation is realized by conjugated gradient algorithm, ${ }^{52}$ which optimizes structure without breaking symmetry. All atoms in the supercell are fully relaxed until the energy difference between the successive steps is less than $10^{-5} \mathrm{eV}$ and the net force on each atom is less than $10^{-2} \mathrm{eV} / \AA$. In addition, maximum pressure on the lattice has been lowered down to 1 kbar. Numerical calculations are carried out by using VASP software. ${ }^{53,54}$

Thermal stability and desorption of specific structures are tested by ab initio, finite temperature molecular dynamics (MD) calculations. A Nosé thermostat was used, and Newtonian equations of motion were integrated through the Verlet algorithm with time steps of $2 \mathrm{fs}$. To determine the energy barrier between the initial and final configurations, we use climbing image nudged elastic band method. ${ }^{55}$ For these calculations, we determined two images, one of them is the initial image of the unrelaxed Gly + graphene system; the other one is the final image of the optimized system. We take into account nine intermediate images with equal intervals between the initial and final states.
The average cohesive energy of Gly is calculated using the expression, $\bar{E}_{\mathrm{c}}=E_{\mathrm{T}}[\mathrm{N}]+E_{\mathrm{T}}[\mathrm{C}]+2 E_{\mathrm{T}}[\mathrm{O}]+5 E_{\mathrm{T}}[\mathrm{H}]-$ $E_{\mathrm{T}}[\mathrm{Gly}]$, in terms of the total energies of constituent atoms and the total energy of Gly. The binding energy of Gly to graphene is calculated by $E_{\mathrm{b}}=E_{\mathrm{T}}$ [graphene $]+E_{\mathrm{T}}[\mathrm{Gly}]-$ $E_{\mathrm{T}}[\mathrm{Gly}+$ graphene $]$ in terms of the total energies of pristine graphene, a free glycine molecule, and glycine adsorbed to graphene, respectively. The binding energy in the physisorption of Gly on pristine graphene is constituted through weak $\mathrm{vdW}$ interaction. The contribution of the chemical interaction to the binding energy in physisorption is determined as the difference of energy between the optimized total energies calculated without and with the vdW correction. If the binding of Gly induces a local reconstruction in graphene, the energy involved with it, $E_{\mathrm{R}}$ is subtracted from the binding energies $E_{\mathrm{b}}$ defined above to redefine the binding energy. The rebonding subsequent to the adsorption of Gly at the edges of nanoribbons is a kind of reconstruction and enhances the binding energy dramatically. The reconstruction or rebonding energy is determined by subtracting the total energy of the substrate calculated for its fixed, reconstructed (or rebonded) geometry from the total energy of Gly + substrate calculated for the fully relaxed reconstructed geometry. Because the binding and dissociation energy of Gly on graphene can change depending on the charge state of the molecule, we calculated pulling energy for different charge states. To this end, starting from the equilibrium configuration, the total energy of charged Gly on graphene is optimized as a function of the distance $h$ between them and the charge in different polarities. At each step, the heights $h$ of at least two atoms of the molecule from the substrate are fixed but the remaining structural parameters are fully optimized.

The bonding is analyzed in terms of the total charge density, $\rho_{\mathrm{T}}$, difference charge densities, $\Delta \rho_{\mathrm{T}}$, obtained by subtracting charge densities of free substrate and Gly, and $\Delta \rho_{\mathrm{A}}$ calculated by subtracting the free atom charge densities from $\rho_{\mathrm{T}}$. The amount of charge transfer between the substrate and Gly is calculated first by subtracting the charge density of the bare, optimized substrate from $\Delta \rho_{\mathrm{T}}$ and then by integrating the remaining charge on Gly, $Z_{\mathrm{Gly}}$. The difference between the value of latter integral and the electronic charge of neutral Gly, $Z_{\mathrm{Gly}}^{\mathrm{o}}$, namely $\Delta Z=Z_{\mathrm{Gly}}-Z_{\mathrm{Gly}}^{\mathrm{o}}$, sets the value of charge transfer. Here, $\Delta Z>0$ indicates electron transfer to Gly from the substrate. 

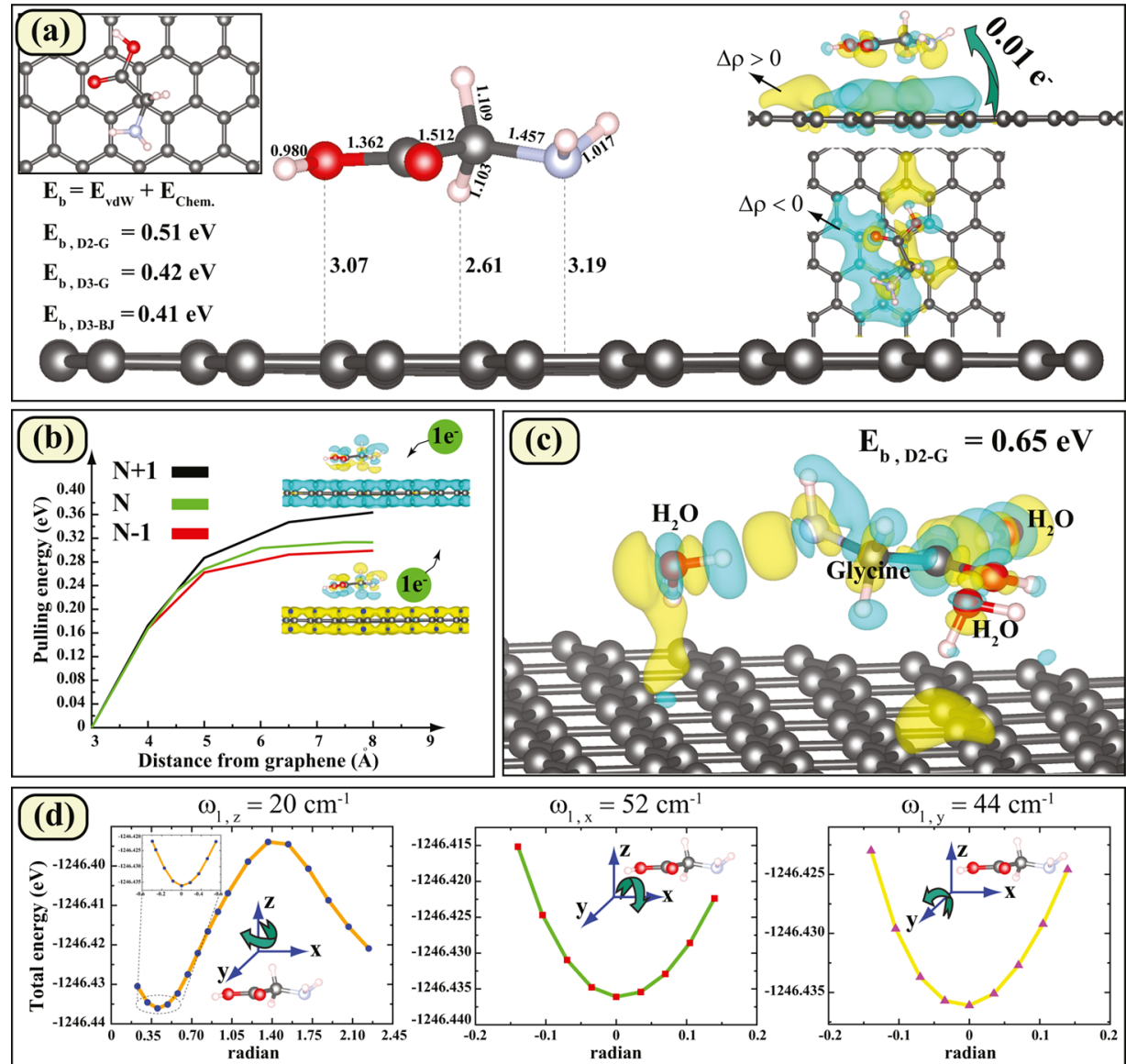

Figure 2. (a) Atomic configuration of Gly physisorbed on pristine graphene. Isosurfaces of difference charge densities $\Delta \rho_{\mathrm{T}}$ on graphene surface and around Gly are shown. Binding energies calculated using different vdW correction methods (D2-G, ${ }^{48} \mathrm{D} 3-\mathrm{G}^{49}{ }^{49}$ and $\mathrm{D} 3-\mathrm{BJ}^{50}$ ) are also given. (b) Variation of energy during the pulling of Gly from the graphene substrate for $N$ (total number of electrons of the neutral system), $N-1$, and $N+1$ electrons of the system. (c) Physisorption of Gly on graphene with coadsorbed $\mathrm{H}_{2} \mathrm{O}$ molecules. (d) Calculated libration frequencies of physisorbed Gly around $x, y$, and $z$ axes.

Free Glycine Molecule. A free glycine molecule, $\mathrm{NH}_{2} \mathrm{CH}_{2} \mathrm{COOH}$, is characterized through its optimized structural parameters and corresponding average cohesive energy $\bar{E}_{\mathcal{c}}$, vibration frequencies, ionization potential (IP), electron affinity (EA), and the distribution of the total electronic charge, as well as electronic energy-level diagram with the highest occupied molecular orbital (HOMO)-lowest unoccupied molecular orbital (LUMO) gap as presented in Figure 1. The calculated vibration frequencies are in fair agreement with experimental and theoretical data. ${ }^{25-30}$ Our calculated bond lengths are comparable with previous theoretical and experimental results. ${ }^{27,30-32}$ Gly is a saturated molecule and hence it has a wide HOMO-LUMO gap of $E_{\mathrm{g}-\mathrm{HL}}=4.59 \mathrm{eV}$ (it becomes $6.20 \mathrm{eV}$ with $\mathrm{HSE}^{33}$ corrections). The present gap value is slightly lower than that of the previous study. ${ }^{14}$ One deduces that HOMO states are derived from $\mathrm{N}$ and $\mathrm{H}$ orbitals, whereas LUMO states originate from $2 \mathrm{p}$ orbitals of $\mathrm{C}$ atom. IP and EA are calculated relative to the optimized neutral, anionic, and cationic glycine molecule. These values are in agreement with those reported in a previous study. ${ }^{34}$ Notably, constituent $\mathrm{N}, \mathrm{C}$, and $\mathrm{O}$ atoms are critical ends of the molecule to form bonds with substrates or to join other molecules.

Binding of Gly To Pristine Graphene. Gly is adsorbed (physisorbed) to graphene via weak vdW interaction. The binding configuration corresponding to the strongest phys- isorption energy, where the molecule takes a lateral orientation relative to the surface of graphene, is described in Figure 2a. The distance between the constituent atoms of Gly and the plane of graphene varies between 2.61 and 3.19 A. The binding energy $E_{\mathrm{b}}$ of this lateral configuration ranges from 0.41 to 0.51 $\mathrm{eV}$ depending on the vdW correction used in calculations (see Computational Details). Binding energies reported earlier vary between 0.30 and $0.72 \mathrm{eV} .^{3,16-18}$ The difference charge density $\Delta \rho_{\mathrm{T}}$ indicates that a small amount of charge is transferred to Gly from graphene. This attributes a minute chemical interaction $(80 \mathrm{meV})$ into the physisorption. Recent works reported $60-90 \mathrm{meV}$ binding energy for Gly on graphene without vdW attraction. ${ }^{14,15}$

The binding of Gly can be manipulated with charge and solvent. The variation of the total energy as one pulls Gly from its equilibrium position on graphene in different charge states is presented in Figure $2 \mathrm{~b}$. When negatively charged, the pulling energy increases. This situation is reversed when the system is positively charged. On the basis of this result, one can conclude that the diffusion (or the migration) and even desorption of Gly on graphene can be monitored by charging. Solvent contribution changes the dielectric of the medium and accordingly the binding energy of an amino acid to the surface becomes stronger with coadsorbed solvent. ${ }^{16,35,36}$ Earlier, it was found that two $\mathrm{H}_{2} \mathrm{O}$ molecules can stabilize a Gly zwitterion by giving at least one vibrational level of the 
potential energy minimum. ${ }^{36}$ In the present study, we examined the stabilization of Gly on graphene surface by optimizing the total energy with three $\mathrm{H}_{2} \mathrm{O}$ molecules initially positioned near the active sites of Gly. We found that the three $\mathrm{H}_{2} \mathrm{O}$ molecules coadsorbed on graphene, as seen in Figure 2c, increase the polarization in Gly; amino nitrogen $\left(\mathrm{NH}_{2}^{+}\right)$ becomes positively polarized, whereas the carboxyl group $\left(\mathrm{COOH}^{-}\right)$is negatively polarized. At the end, the binding of zwitterionic glycine to graphene can be slightly stronger $(0.14$ $\mathrm{eV}$ more) in the presence of coadsorbed $\mathrm{H}_{2} \mathrm{O}$ molecules at the close proximity of Gly.

Gly physisorbed to graphene can also attain libration frequencies for its rotational vibration as whole around three axes. Three libration frequencies of physisorbed Gly are calculated in Figure 2d. Even if they are soft, they can be measured. In particular, when the physisorption configuration varies or a foreign atom or small molecule is attached to Gly, the libration frequencies undergo a change, which can be detected easily. ${ }^{25,37}$ Once the above arguments are extended to other amino acids, their libration frequencies appear to be an important feature (fingerprint) to characterize a particular molecule. These energy versus radian graphs also prove that Gly prefers physisorbed with parallel orientation on graphene.

Pinning of Gly at Defect Sites. Weak vdW interaction between Gly and graphene cannot provide the fixation of the molecule on the substrate, rather Gly would diffuse on the substrate or even desorb from graphene under thermal excitations. Therefore, strong binding of Gly is desired for its characterization and self-assembly. In pristine graphene, three $\mathrm{sp}^{2}$-hybrid orbitals oozing from each $\mathrm{C}$ atom are saturated and formed strong $\mathrm{C}-\mathrm{C}$ bonds, which render inertness. Maintaining several advantages of graphene being an ideal biointerface, Gly can be pinned if it is bound to defect sites, such as single vacancy $(\mathrm{V})$, trivacancy $(3 \mathrm{~V})$, or edges of graphene, and can possess low-coordinated $\mathrm{C}$ atoms with unsaturated $\mathrm{sp}^{2}$-bonds.

Gly Bound To the Edge of Ribbons. Armchair N-AGNR or zigzag $N$-ZGNR nanoribbons, with $N$ being the number of $\mathrm{C}$ atoms in the unit cell, both are quasi one-dimensional (1D) and both possess a row of twofold coordinated $\mathrm{C}$ atoms with one dangling bond at their edges. By attaching one $\mathrm{C}$ atom to each twofold coordinated edge atoms, one constructs a Klein edge (KE) defect, as also observed experimentally. ${ }^{38}$ These nanoribbons are generally planar; the two edges can couple when they are narrow (or $N$ is small).

If Glys approach both edges of $12-\mathrm{ZGNR}$ with their $\mathrm{NH}_{2}$ ends, one $\mathrm{H}$ atom of each Gly dissociates and subsequently is rebound to a twofold coordinated $\mathrm{C}$ atom. Remaining parts $\left(\mathrm{NHCH}_{2} \mathrm{COOH}\right)$ are bound to nearest low-coordinated $\mathrm{C}$ atoms as shown in Figure 3a. Under these circumstances, the binding energy is $4.85 \mathrm{eV}$ for each Gly, which also includes dissociation of an $\mathrm{H}$ atom from $\mathrm{NH}_{2}$ and its rebonding to the nanoribbon. The strong binding of Gly to both edges of 12ZGNR and saturation of $\mathrm{C}$ atoms at each edge causes the nanoribbon to bow, whereby a strain energy can be associated with bowing. In contrast, extended KEs can significantly increase the reactivity of nanoribbons. Two types of KEs, $\mathrm{KE}_{1}$ and $\mathrm{KE}_{2}$ are distinguished in Figure 3. In Figure 3b, Gly molecules are strongly bound to $\mathrm{KE}_{1}$ from both edges with the nearest twofold coordinated $\mathrm{C}$ atoms also saturated by the dissociated $\mathrm{H}$ atoms. In Figure $3 \mathrm{c}$, neither the optimized atomic structure of 11-AGNR is affected significantly upon the adsorption of Gly nor its $\mathrm{H}$ atom is dissociated. The situation is rather different when Gly molecules are adsorbed to both
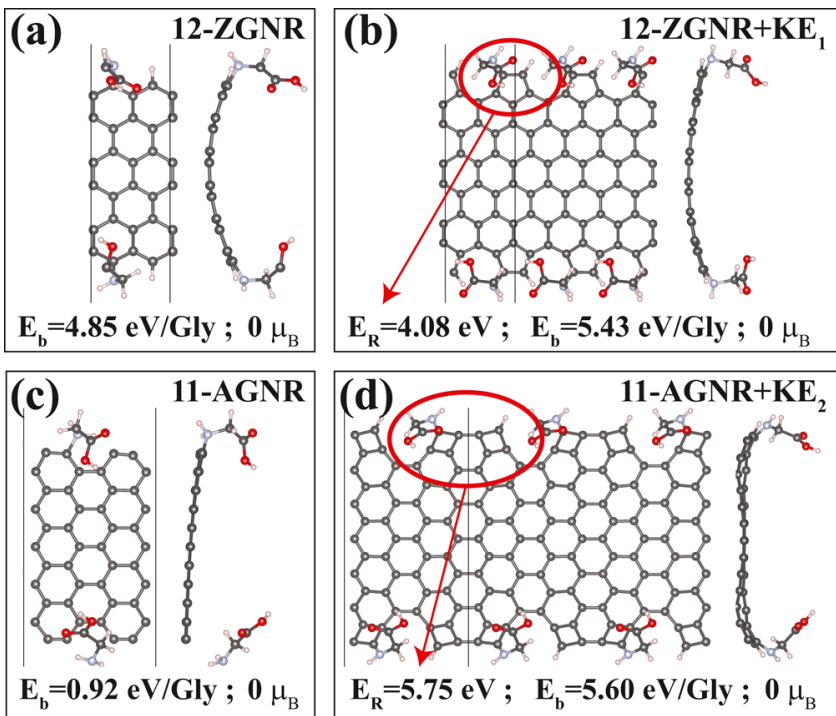

Figure 3. Atomic configurations and binding energies $\left(E_{\mathrm{b}}\right)$ of Glys which are bound to various nanoribbons of graphene through their $\mathrm{NH}_{2}$ ends: (a) Glys are attached to both edges of zigzag nanoribbon 12-ZGNR. (b) Glys are bound to Klein edged zigzag nanoribbon, 12$\mathrm{ZGNR}+\mathrm{KE}_{1}$. (c) Glys are bound to the armchair nanoribbon, 11AGNR. (d) Glys are bound to Klein edged armchair nanoribbon, 11$\mathrm{AGNR}+\mathrm{KE}_{2}$.

edges of 11-AGNR $+\mathrm{KE}_{2}$. The adsorption of Gly molecules to both edges is followed by the saturation of $\mathrm{C}$ atoms by $\mathrm{H}$ atoms subsequent to their desorption from Gly, as well as massive reconstruction and bowing of the nanoribbon. Accordingly, the reconstruction and binding energies are calculated to be rather high. Notably, Glys form rows at the edges and significantly modify the electronic structure of bare nanoribbons. In particular, additional bands of the states located at the edges originating from the ordered rows of Glys can occur. It should be noted that the adsorption of Glys at the edges on the same side complies with possible applications because the nanoribbon is normally supported by a substrate from one side, leaving the other side free for adsorption. We found that the ribbon has been bowed following the adsorption, even if Gly molecules are initially placed in the same plane of the nanoribbon. However, one can expect that the planar geometry of the nanoribbon would be maintained if Gly molecules were adsorbed at alternative sides.

Gly Bound To a Trivacancy. Trivacancy (3V) in graphene is normally reconstructed, where surrounding $\mathrm{C}$ atoms become rebound to minimize the number of unsaturated $\mathrm{sp}^{2}$-bonds. Eventually, there remain only one twofold coordinated $\mathrm{C}$ atom with one unsaturated (dangling) $\mathrm{sp}^{2}$-bond, whereas the rest become threefold coordinated. Graphene $+3 \mathrm{~V}$ treated by an $(8 \times 8)$ supercell is a magnetic metal with $\mu=1.0 \mu_{\mathrm{B}}$ per cell. A Gly attains diverse binding configurations at the $3 \mathrm{~V}$ site with different binding energies as shown in Figure 4a,b. Most of these configurations have weak bonding with $E_{\mathrm{b}}<0.6 \mathrm{eV}$, except $C_{5}$ configuration, which involves a strong chemical binding through the unsaturated $\mathrm{sp}^{2}$-bond with $E_{\mathrm{b}}=1.93 \mathrm{eV}$. The graphene $+3 \mathrm{~V}$ system undergoes a dramatic change after the chemisorption of Gly in $C_{5}$ configuration as described in Figure $4 \mathrm{c}$; the whole system changes into a nonmagnetic semiconductor with a direct band gap of $0.263 \mathrm{eV}$, followed by the changes of energy-level diagram of Gly, as well as the peak positions of density of states. The nonmagnetic state of the 

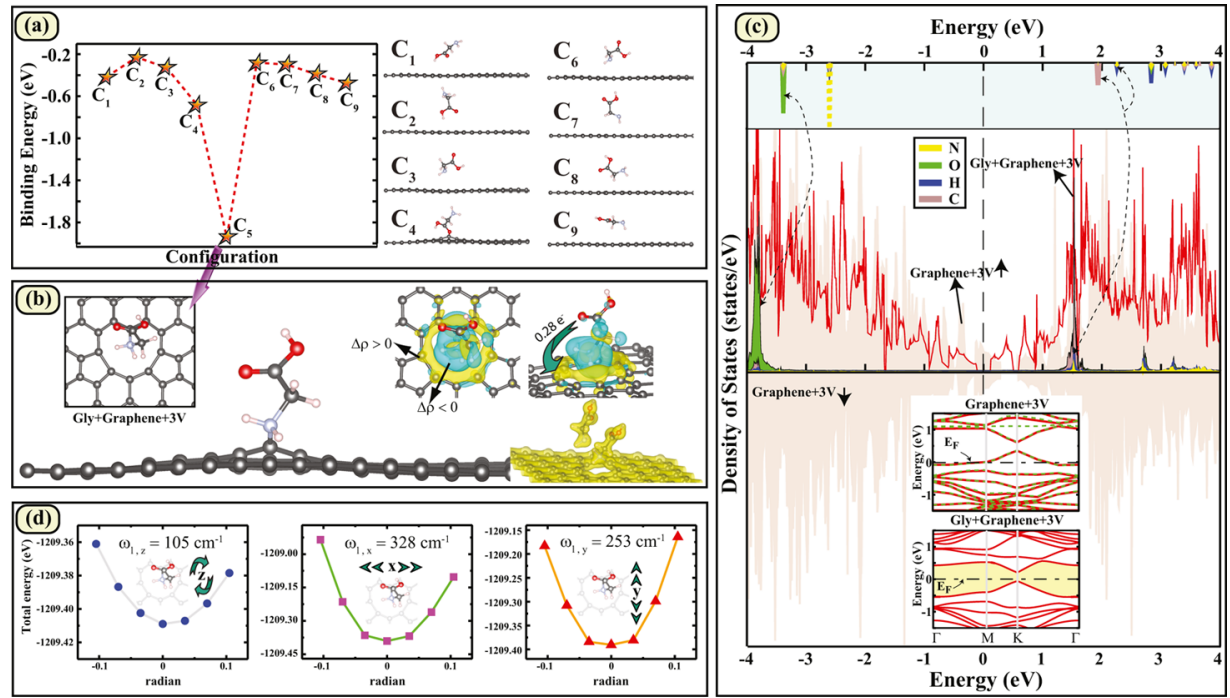

Figure 4. (a) Various configurations of Gly bound to the trivacancy $(3 \mathrm{~V})$ formed in each $(8 \times 8)$ supercell graphene (graphene $+3 \mathrm{~V})$ and resulting binding energies. (b) The configuration specified as $C_{5}$ has the strongest binding and hence pins Gly on graphene. Difference charge density, $\Delta \rho_{\mathrm{T}}$, isosurfaces clarify electron exchange between a molecule and graphene to construct a chemical bond. Bottom-right corner is for coadsorption of two Glys. (c) Top: Distribution of the discrete states of free Gly. Bottom: Spin-polarized state densities of graphene $+3 \mathrm{~V}$ are shown by gray background. Spin unpolarized total and atom projected densities of states of Gly + graphene $+3 \mathrm{~V}$ are also shown. The state of an $\mathrm{N}$ atom at the $-2.6 \mathrm{eV}$ disappeared after its binding to graphene $+3 \mathrm{~V}$. The energy band structures of graphene $+3 \mathrm{~V}$ and Gly + graphene $+3 \mathrm{~V}$ are shown by insets. (d) Libration frequencies of Gly adsorbed to trivacancy $(3 \mathrm{~V})$ site of graphene in $C_{5}$ configuration. Libration frequencies are calculated along $x, y$, and $z$ directions.
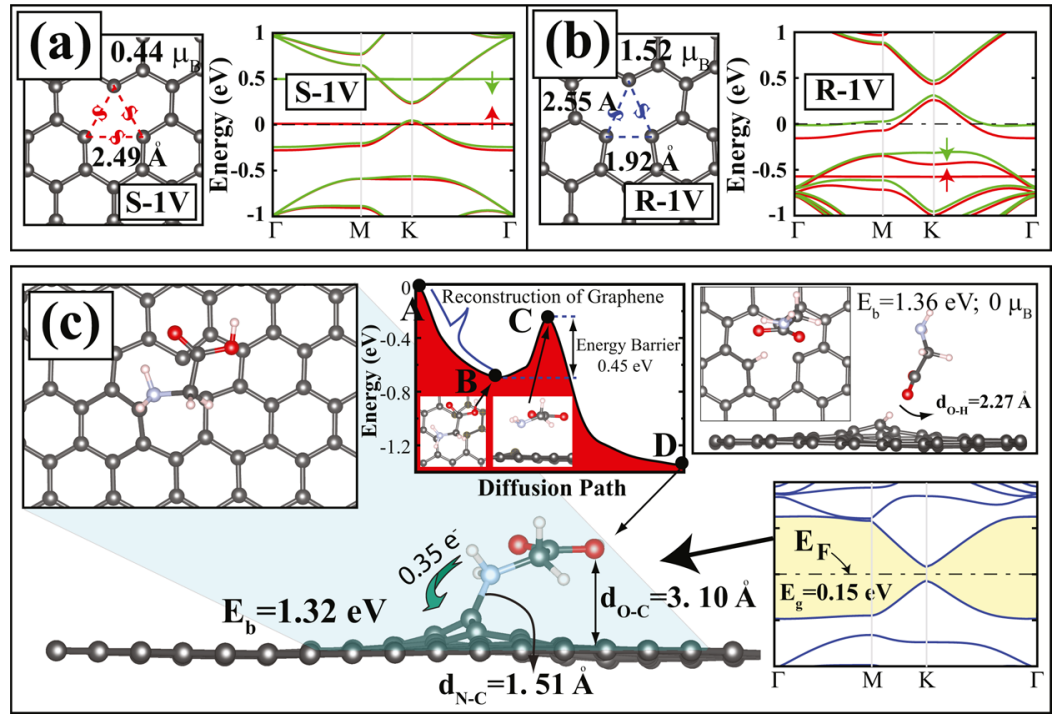

Figure 5. (a) Atomic configuration, structural parameters, magnetic moment, and energy band structure of single symmetric (unreconstructed) vacancy formed in an $(8 \times 8)$ supercell of graphene, $(\mathrm{S}-1 \mathrm{~V})$. (b) Same for reconstructed single vacancy (R-1V). (c) Top and side views of the atomic configuration of Gly adsorbed to S-1V at one of two-folded active site from its nitrogen end with the binding energy $E_{\mathrm{b}}=1.32 \mathrm{eV}$. Upon adsorption, the metallic and magnetic S-1V system is transformed into a nonmagnetic, semiconductor with $0.15 \mathrm{eV}$ band gap. Up-right corner describes a different but weakly bound Gly attached to the defect site from its $\mathrm{COOH}$ side. One of $\mathrm{H}$ atoms is dissociated and subsequently bound to one $\mathrm{C}$ atom of graphene; the remaining part of Gly interacts weakly and hence raises on the surface.

system is confirmed by examining the difference of the densities of states of spin-up and spin-down states for all energies. These are metal-insulator and magnetic-nonmagnetic transitions, which are critical changes to be utilized in sensor applications. Notably, a second Gly placed at $3 \mathrm{~V}$ at was repelled by the Gly already adsorbed in $C_{5}$ configuration. This shows that $3 \mathrm{~V}$ can bind only one Gly strongly.

The libration frequencies of Gly adsorbed to graphene $+3 \mathrm{~V}$ with $C_{5}$ configuration became 4-5 times higher as compared to those of Gly physisorbed to pristine graphene. In Figure $4 \mathrm{~d}$, libration frequencies $\omega_{\mathrm{L}}$, calculated along $x, y$, and $z$ axes indicate significant restoring forces to maintain the adsorbed molecules in ordered geometry, which is essential for their selfassembly behaviors. Even if the thermal stability of the adsorbed Gly of $C_{5}$ configuration at $300 \mathrm{~K}$ is assured by performing ab initio $\mathrm{MD}$ simulations of $2 \mathrm{ps}$, at higher temperatures $T>400 \mathrm{~K}$, the $\mathrm{COOH}$ group is dissociated from the system.

Gly Bound To a Single Vacancy. The adsorption of Gly at the site of a single (isolated) vacancy (1V) is also mimicked 


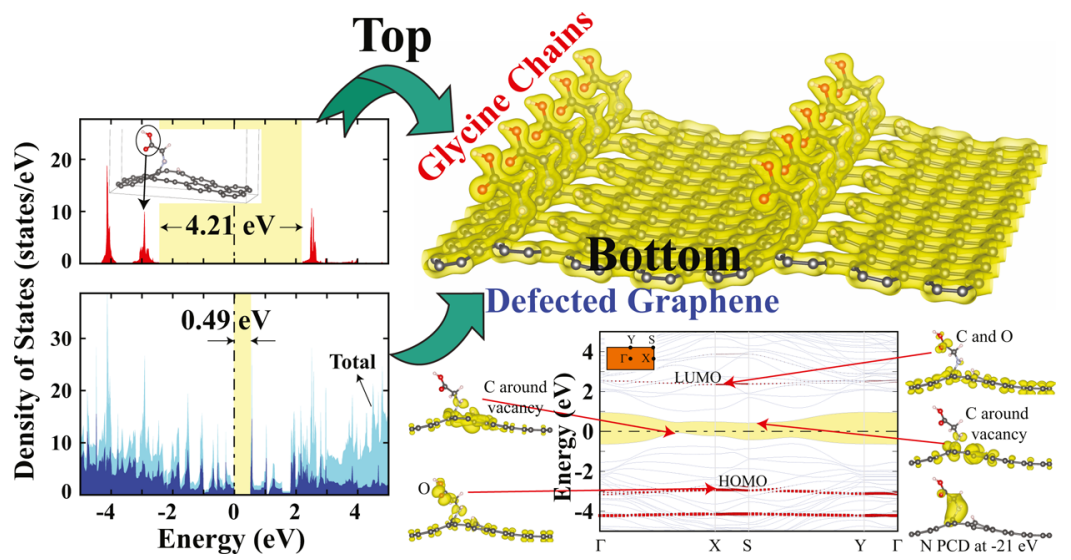

Figure 6. Top right-hand corner: The perspective view of Glys self-assembled on a graphene with patterned vacancies forming quasi 1D stripes. Left panel: Density of states projected to Gly overlayer and graphene. The band gaps are shaded and indicate the insulator-semiconductor junction. Zero of energy is set at the Fermi energy, $E_{\mathrm{F}}$. Bottom right-hand corner: Energy band structure of the junction. The narrowest band gap at the graphene side is shaded; the band edges at the Gly overlayer side are shown by the red dotted lines. Band-projected charge density isosurfaces of the bands at the edges of the gap and nitrogen band at $-21 \mathrm{eV}$ below $E_{\mathrm{F}}$.

using an $(8 \times 8)$ supercell. Two types of single vacancy in graphene, that is, symmetric ( $\mathrm{S}-1 \mathrm{~V})$ and reconstructed vacancy (R-1V) observed in graphene, ${ }^{39}$ are shown in Figure 5a,b. The calculated energy band structures demonstrate that both vacancies are magnetic metals. When placed near $1 \mathrm{~V}$ in graphene, a Gly is adsorbed to a twofold coordinated $\mathrm{C}$ atom surrounding $1 \mathrm{~V}$ with $E_{\mathrm{b}}=1.32 \mathrm{eV}$ by donating electronic charge of $-0.35 e$ to graphene from its nitrogen end as shown in Figure 5c. Results of NEB calculations starting from the parallel-oriented Gly, $2.4 \AA$ above S-1V (point A) are presented in the inset in Figure 5c. Initially, $S-1 V$ first transforms into R-1V (point B), and then, overcoming a barrier of $0.45 \mathrm{eV}$ (point $\mathrm{C}$ ), it eventually becomes adsorbed to R-1V as shown by subinsets. This energy barrier is related to the raising of the unsaturated $\mathrm{C}$ atom. Like $3 \mathrm{~V}$, upon adsorption, bare graphene $+1 \mathrm{~V}$, which is a magnetic metal, changes into a nonmagnetic insulator with $0.15 \mathrm{eV}$ band gap. This is an important effect and can easily be detected through conductance measurements and also can be exploited as a detector of Gly. Another adsorption configuration of Gly, in which one $\mathrm{H}$ atom dissociates from the $\mathrm{O}-\mathrm{H}$ bond and subsequently saturates one $\mathrm{C}$ atom at the defect site with the remaining part of Gly being physisorbed is shown in the inset in Figure 5c. The dissociation of an $\mathrm{H}$ atom from Gly occurs because Gly is initially placed close to the defect site to generate a strong interaction. The weakly bound part, which raises on the surface, has only weak interaction with graphene.

Self-Assembly of Gly: Junction Formation. A Gly adsorbed at an isolated defect site of graphene has a welldefined configuration/orientation, which is maintained by the restoring forces driven by its chemical bond. These restoring forces become apparent by significantly high libration frequencies. This behavior is identified as a self-assembly, which is essential for producing ordered organic films on defect-patterned graphene to achieve a composite material from amino acids. Recently, significant progress has been made to create patterned defects on pristine graphene. The fabrication of large graphene sheets with a high-density array of nanoscale holes, called nanomeshes ${ }^{40}$ has been realized. The formation of $1 \mathrm{D}$ periodic SW defects on graphene has been reported. ${ }^{41,42}$ Theoretical studies have predicted critical effects of periodically repeating holes on electronic properties. ${ }^{43,44}$
1D-Patterned Adsorption. Quasi 1D, defect-patterned adsorption of Gly on graphene is considered by using $1 \mathrm{~V}$ created in a cell of $4.94 \AA \times 17.10 \AA$. To allow more variational freedom, a supercell is taken to contain two adjacent $1 \mathrm{~V}$, whereby rows of $1 \mathrm{~V}$ are formed in graphene along the $x$-axis. When free of Gly, this defected substrate is a magnetic metal. Gly molecules approaching to defected graphene in different orientations and through different ends can trigger reconstruction in the substrate. However, when attached to a twofold coordinated $\mathrm{C}$ atom of the defect, Gly can form strong chemical bond and cause magnetic and metallic graphene to transform to a nonmagnetic, narrow band gap semiconductor similar to other defect sites. Despite their preset initial configuration, all Glys are reoriented and have the same final configuration but different from the initial one. As a further test of the self-assembly character, the initial configuration of one of four Glys in a larger supercell was changed randomly from the others; nonetheless four of them attained the same configuration upon optimization. This situation provides a strong evidence for the self-assembly of adsorbed Glys.

As shown in Figure 6 (top right-hand corner), selfassembled Glys formed rows (stripes) and hence construct an organic overlayer on graphene. Because of the strong binding of Gly, this organic overlayer persists at room temperature. This self-assembled system can be viewed as a composite material realized through the junction (heterostructure) of Gly overlayer and defected graphene. The density of states projected to the top of the Gly overlayer has a large band gap of $4.21 \mathrm{eV}$, whereas the band gap revealed from that projected to graphene is rather narrow in Figure 6 (left panel). In principle, it is an insulator-semiconductor junction with well-defined band alignment and exhibits side-dependent, bilateral electronic and optical properties. Calculated bands of the composite system and charge density isosurfaces of states at the band edges in Figure 6 (bottom right-hand corner) demonstrate the side-dependent electronic structure. HOMO and LUMO states are hybridized with graphene bands. Relatively low-dispersion HOMO and LUMO bands imply weak coupling between the atoms of self-assembled Glys. 


\section{CONCLUSIONS}

Despite the fact that the interaction between Gly and pristine graphene is weak vdW attraction, twofold coordinated $\mathrm{C}$ atoms at the edges of nanoribbons or single- and triple-vacancy sites act as active sites to exchange significant charge with the molecule. This way, a chemical bonding is set between Gly and the substrate, which pins the molecule with a significant binding energy. Pinning of this amino acid on the surface of graphene enables its characterization and is an important step toward the synthesis of new peptides. We note that in all processes outlined above, Gly is generally adsorbed to lowcoordinated $\mathrm{C}$ atoms with significant binding from its $\mathrm{NH}_{2}$ end; whereas the bonding with graphene at defect sites from other ends is relatively weaker. When bound to the defect site, Gly induces a metal-insulator transition, where the magnetic and metallic substrate changes to a narrow band gap semiconductor. However, this transition does not occur if Gly was bound through weak vdW interaction.

Through pinning at the vacancy site, a Gly attains significant libration frequencies, where the rotation of the molecule as a whole induces restoring force. Accordingly, these restoring forces are strong enough to keep the adsorbed Gly at a welldefined configuration.

Glys self-assembled on the defect-patterned graphene can form an organic thin film (overlayer) with desired decoration and dimensionality. In this paper, we studied quasi 1D stripes of Gly on graphene forming a junction, with one side being a wide band gap insulator and the other side being a narrow band gap semiconductor.

Finally, the semiconducting graphene side can be metallized by the chemisorption of alkali metals to graphene between two self-assembled Gly rows. This way a semiconductor-metal junction and hence a Schottky barrier can be attained. It should be noted that not only insulating $1 \mathrm{D}$ rows but also $2 \mathrm{D}$ meshes of diverse size and geometry can be realized from selfassembled Glys or other amino acids on graphene or on similar monolayer structures to construct bio-2D material heterostructures with novel functionalities.

\section{AUTHOR INFORMATION}

\section{Corresponding Authors}

*E-mail: ethem.akturk@adu.edu.tr (E.A.).

*E-mail: ciraci@fen.bilkent.edu.tr. Phone: +9025621308351894. Fax: +902562135379 (S.C.).

ORCID $\odot$

E. Aktürk: 0000-0002-1615-7841

Notes

The authors declare no competing financial interest.

\section{ACKNOWLEDGMENTS}

Authors acknowledges useful discussion with Dr. Urartu Şeker and Yelda Kadioglu. This research was supported in part by TÜBITAK (The Scientific \& Technological Research Council of Turkey) through TR-Grid e-Infrastructure Project, part of the calculations have been carried out at ULAKBIM computer center. S.C. acknowledges the financial support from the Academy of Science of Turkey TÜBA.

\section{REFERENCES}

(1) Novoselov, K. S.; Geim, A. K.; Morozov, S. V.; Jiang, D.; Katsnelson, M. I.; Grigorieva, I. V.; Dubonos, S. V.; Firsov, A. A. Two- dimensional gas of massless Dirac fermions in graphene. Nature 2005, 438, 197-200.

(2) Schedin, F.; Geim, A. K.; Morozov, S. V.; Hill, E. W.; Blake, P.; Katsnelson, M. I.; Novoselov, K. S. Detection of individual gas molecules adsorbed on graphene. Nat. Mater. 2007, 6, 652-655.

(3) Dragneva, N.; Floriano, W. B.; Stauffer, D.; Mawhinney, R. C.; Fanchini, G.; Rubel, O. Favorable adsorption of capped amino acids on graphene substrate driven by desolvation effect. J. Chem. Phys. 2013, 139, 174711.

(4) Dong, X.; Cheng, J.; Li, J.; Wang, Y. Graphene as a Novel Matrix for the Analysis of Small Molecules by MALDI-TOF MS. Anal. Chem. 2010, 82, 6208-6214.

(5) Liang, L.; Shen, J.-W.; Zhang, Z.; Wang, Q. DNA sequencing by two-dimensional materials: As theoretical modeling meets experiments. Biosens. Bioelectron. 2017, 89, 280-292.

(6) Wang, Q.; Wang, M.-h.; Wang, K.-f.; Liu, Y.; Zhang, H.-p.; Lu, X.; Zhang, X.-d. Computer simulation of biomolecule-biomaterial interactions at surfaces and interfaces. Biomed. Mater. 2015, 10, 032001.

(7) Cazorla, C. Ab initio study of the binding of collagen amino acids to graphene and $\mathrm{A}$-doped $(\mathrm{A}=\mathrm{H}, \mathrm{Ca})$ graphene. Thin Solid Films 2010, 518, 6951-6961.

(8) Ma, F.; Zhang, Z.; Jia, H.; Liu, X.; Hao, Y.; Xu, B. Adsorption of cysteine molecule on intrinsic and Pt-doped graphene: A firstprinciple study. J. Mol. Struct.: THEOCHEM 2010, 955, 134-139.

(9) Wang, Y.; Li, Y.; Tang, L.; Lu, J.; Li, J. Application of graphenemodified electrode for selective detection of dopamine. Electrochem. Commun. 2009, 11, 889-892.

(10) Rajesh, C.; Majumder, C.; Mizuseki, H.; Kawazoe, Y. A theoretical study on the interaction of aromatic amino acids with graphene and single walled carbon nanotube. J. Chem. Phys. 2009, $130,124911$.

(11) Ortiz-Medina, J.; López-Urías, F.; Terrones, H.; RodríguezMacías, F. J.; Endo, M.; Terrones, M. Differential Response of Doped/Defective Graphene and Dopamine to Electric Fields: A Density Functional Theory Study. J. Phys. Chem. C 2015, 119, 13972-13978.

(12) Davies, N. P.; Wilson, M.; Natarajan, K.; Sun, Y.; MacPherson, L.; Brundler, M.-A.; Arvanitis, T. N.; Grundy, R. G.; Peet, A. C. Noninvasive detection of glycine as a biomarker of malignancy in childhood brain tumours using in-vivo1H MRS at 1.5 Tesla confirmed by ex-vivo high-resolution magic-angle spinning NMR. NMR Biomed. 2010, 23, 80-87.

(13) Kinoshita, Y.; Kajiwara, H.; Yokota, A.; Koga, Y. Proton Magnetic Resonance Spectroscopy of Brain TumorsAn In Vitro Study. Neurosurgery 1994, 35, 606-614.

(14) Naderi, F.; Karami, A.; Naderi, B. The Interaction between Glycine and Carbon Nanostructure. Org. Chem. J. 2010, 1, 44-49.

(15) Wang, M.-h.; Guo, Y.-n.; Wang, Q.; Zhang, X.-s.-y.; Huang, J.-j.; Lu, X.; Wang, K.-f.; Zhang, H.-p.; Leng, Y. Density functional theory study of interactions between glycine and $\mathrm{TiO} 2 /$ graphene nanocomposites. Chem. Phys. Lett. 2014, 599, 86-91.

(16) Tavassoli Larijani, H.; Darvish Ganji, M.; Jahanshahi, M. Trends of amino acid adsorption onto graphene and graphene oxide surfaces: a dispersion corrected DFT study. RSC Adv. 2015, 5, 92843-92857.

(17) Larijani, H. T.; Jahanshahi, M.; Ganji, M. D.; Kiani, M. H. Computational studies on the interactions of glycine amino acid with graphene, h-BN and h-SiC monolayers. Phys. Chem. Chem. Phys. 2017, 19, $1896-1908$.

(18) Chen, X.; Gao, P.; Guo, L.; Zhang, S. Graphdiyne as a promising material for detecting amino acids. Sci. Rep. 2015, 5, 16720.

(19) Topsakal, M.; Şahin, H.; Ciraci, S. Graphene coatings: An efficient protection from oxidation. Phys. Rev. B: Condens. Matter Mater. Phys. 2012, 85, 155445.

(20) Al-Aqtash, N.; Vasiliev, I. Ab Initio Study of Carboxylated Graphene. J. Phys. Chem. C 2009, 113, 12970-12975. 
(21) Ghaderi, N.; Peressi, M. First-Principle Study of Hydroxyl Functional Groups on Pristine, Defected Graphene, and Graphene Epoxide. J. Phys. Chem. C 2010, 114, 21625-21630.

(22) Denis, P. A.; Iribarne, F. Comparative Study of Defect Reactivity in Graphene. J. Phys. Chem. C 2013, 117, 19048-19055.

(23) Gürel, H. H.; Özçelik, V. O.; Ciraci, S. Dissociative Adsorption of Molecules on Graphene and Silicene. J. Phys. Chem. C 2014, 118, 27574-27582.

(24) Gao, F.; Gao, S. Controlling magnetic transition of monovacancy graphene by shear distortion. Sci. Rep. 2017, 7, 1792.

(25) Kumar, S.; Rai, A. K.; Singh, V. B.; Rai, S. B. Vibrational spectrum of glycine molecule. Spectrochim. Acta, Part A 2005, 61, 2741-2746.

(26) Stenback, H. On the Raman spectra of solid natural $\alpha$-glycine and solid $15 \mathrm{~N}$-substituted $\alpha$-glycine. J. Raman Spectrosc. 1976, 5, 4955 .

(27) López-Navarrete, J. T.; Quirante, J. J.; Ramírez, F. J. Harmonic force field for the glycine molecule by semiempirical methods. J. Mol. Struct. 1992, 268, 249-261.

(28) Lafosse, A.; Bertin, M.; Domaracka, A.; Pliszka, D.; Illenberger, E.; Azria, R. Reactivity induced at $25 \mathrm{~K}$ by low-energy electron irradiation of condensed NH3-CH3COOD (1: 1) mixture. Phys. Chem. Chem. Phys. 2006, 8, 5564-5568.

(29) Shi, Y.; Wang, L. Collective vibrational spectra of $\alpha$ - and $\gamma$ glycine studied by terahertz and Raman spectroscopy. J. Phys. D: Appl. Phys. 2005, 38, 3741.

(30) Barone, V.; Adamo, C.; Lelj, F. Conformational behavior of gaseous glycine by a density functional approach. J. Chem. Phys. 1995, 102, 364.

(31) Kaschner, R.; Hohl, D. Density Functional Theory and Biomolecules: A Study of Glycine, Alanine, and Their Oligopeptides. J. Phys. Chem. A 1998, 102, 5111-5116.

(32) Kumar, S.; Rai, A. K.; Singh, V. B.; Rai, S. B. Vibrational spectrum of glycine molecule. Spectrochim. Acta, Part A 2005, 61, 2741-2746.

(33) Heyd, J.; Scuseria, G. E.; Ernzerhof, M. Hybrid Functionals Based on a Screened Coulomb Potential. J. Chem. Phys. 2003, 118, $8207-8215$

(34) Maul, R.; Preuss, M.; Ortmann, F.; Hannewald, K.; Bechstedt, F. Electronic Excitations of Glycine, Alanine, and Cysteine Conformers from First-Principles Calculations. J. Phys. Chem. A 2007, 111, 4370-4377.

(35) Dragneva, N.; Floriano, W. B.; Stauffer, D.; Mawhinney, R. C.; Fanchini, G.; Rubel, O. Favorable adsorption of capped amino acids on graphene substrate driven by desolvation effect. J. Chem. Phys. 2013, 139, 174711.

(36) Jensen, J. H.; Gordon, M. S. On the Number of Water Molecules Necessary To Stabilize the Glycine Zwitterion. J. Am. Chem. Soc. 1995, 117, 8159-8170.

(37) Aktürk, O. Ü.; Aktürk, E.; Gürel, H. H.; Ciraci, S. Tunable dynamics of a flake on graphene: Libration frequency. Phys. Rev. B 2017, 95, 125413.

(38) He, K.; Robertson, A. W.; Lee, S.; Yoon, E.; Lee, G.-D.; Warner, J. H. Extended Klein Edges in Graphene. ACS Nano 2014, 8, $12272-12279$.

(39) Robertson, A. W.; Montanari, B.; He, K.; Allen, C. S.; Wu, Y. A.; Harrison, N. M.; Kirkland, A. I.; Warner, J. H. Structural Reconstruction of the Graphene Monovacancy. ACS Nano 2013, 7, 4495-4502.

(40) Bai, J.; Zhong, X.; Jiang, S.; Huang, Y.; Duan, X. Graphene nanomesh. Nat. Nanotechnol. 2010, 5, 190-194.

(41) Lahiri, J.; Lin, Y.; Bozkurt, P.; Oleynik, I. I.; Batzill, M. An extended defect in graphene as a metallic wire. Nat. Nanotechnol. 2010, 5, 326-329.

(42) Balog, R.; Jørgensen, B.; Nilsson, L.; Andersen, M.; Rienks, E.; Bianchi, M.; Fanetti, M.; Lægsgaard, E.; Baraldi, S.; Lizzit, S.; et al. Bandgap opening in graphene induced by patterned hydrogen adsorption. Nat. Mater. 2010, 9, 315-319.
(43) Topsakal, M.; Aktürk, E.; Sevinçli, H.; Ciraci, S. First-principles approach to monitoring the band gap and magnetic state of a graphene nanoribbon via its vacancies. Phys. Rev. B: Condens. Matter Mater. Phys. 2008, 78, 235435.

(44) Sahin, H.; Ciraci, S. Structural, mechanical, and electronic properties of defect-patterned graphene nanomesh from firstprinciples. Phys. Rev. B: Condens. Matter Mater. Phys. 2011, 84, 035452.

(45) Blöchl, P. E. Projector augmented-wave method. Phys. Rev. B: Condens. Matter Mater. Phys. 1994, 50, 17953-17979.

(46) Kresse, G.; Joubert, D. From ultrasoft pseudopotentials to the projector augmented-wave method. Phys. Rev. B: Condens. Matter Mater. Phys. 1999, 59, 1758-1775.

(47) Perdew, J. P.; Burke, K.; Ernzerhof, M. Generalized Gradient Approximation Made Simple. Phys. Rev. Lett. 1996, 77, 3865-3868.

(48) Grimme, S. Semiempirical GGA-type density functional constructed with a long-range dispersion correction. J. Comput. Chem. 2006, 27, 1787-1799.

(49) Moellmann, J.; Grimme, S. DFT-D3 Study of Some Molecular Crystals. J. Phys. Chem. C 2014, 118, 7615-7621.

(50) Johnson, E. R.; Becke, A. D. Van der Waals interactions from the exchange hole dipole moment: Application to bio-organic benchmark systems. Chem. Phys. Lett. 2006, 432, 600-603.

(51) Monkhorst, H. J.; Pack, J. D. Special points for Brillouin-zone integrations. Phys. Rev. B: Solid State 1976, 13, 5188-5192.

(52) Schlegel, H. B. Optimization of equilibrium geometries and transition structures. J. Comput. Chem. 1982, 3, 214-218.

(53) Kresse, G.; Furthmüller, J. Efficient iterative schemes for $a b$ initio total-energy calculations using a plane-wave basis set. Phys. Rev. B: Condens. Matter Mater. Phys. 1996, 54, 11169-11186.

(54) Kresse, G.; Furthmüller, J. Efficiency of ab-initio total energy calculations for metals and semiconductors using a plane-wave basis set. Comput. Mater. Sci. 1996, 6, 15-50.

(55) Henkelman, G.; Uberuaga, B. P.; Jónsson, H. A Climbing Image Nudged Elastic Band Method for Finding Saddle Points and Minimum Energy Paths. J. Chem. Phys. 2000, 113, 9901. 